\title{
Original
}

\section{Changes of Adrenocortical Function and the Ovarian Contribution with Age}

\author{
Hiroshi Saito, Tatsuya Akamatsu and Takumi Yanainara
}

\begin{abstract}
Dissociation of adrenal cortisol and androgen secretion occurs in elderly men and women. The postmenopausal ovary produces androgens; however, previous reports regarding adrenocortical function in postmenopausal women did not exclude the ovarian contribution to peripheral steroid levels. In the present study, changes in the ovarian contribution to adrenocortical function with age were determined by measuring peripheral concentrations of $\mathrm{C}_{19}$ and $\mathrm{C}_{21}$ steroids before and 2 weeks after ovariectomy. The rapid adrenocorticotropic hormone (ACTH) stimulation test (tetracosactide acetate, $0.25 \mathrm{mg}$ i.v.) was performed, and the age-related difference in the response of steroid levels to ACTH stimulation was also determined in ovariectomized reproductive-age (mean age, 33.0 years, $n=6$ ) and postmenopausal women (mean age, 63.3 years, $\mathrm{n}=9$ ). After ovariectomy, the concentrations of pregnenolone, $17 \alpha$-hydroxypregnenolone, progesterone, 17 $\alpha$-hydroxyprogesterone, dehydroepiandrosterone, and androstenedione in reproductive-age women decreased significantly to the levels in postmenopausal women. On the other hand, no significant changes in steroid levels before and after ovariectomy were observed in postmenopausal women. When changes in steroid levels in response to ACTH were compared, significant age-related reductions of dehydroepiandrosterone, and androstenedione were observed with age but $\Delta 4$ and $\Delta 5 \mathrm{C}_{21}$ steroid levels in response to ACTH were preserved. These results suggest that $\mathbf{C}_{17-20}$ lyase activity decreases but $17 \alpha$-hydroxylase is maintained for preservation of cortisol in response to ACTH. It is known that $\mathrm{P} 450 \mathrm{c} 17$ is a single enzyme that catalyzes activity of both $17 \alpha$-hydroxylase and $\mathrm{C}_{17-20}$ lyase. Therefore, changes in adrenal $\mathrm{C}_{17-20}$ lyase activity with age might affect adrenal androgen production.
\end{abstract}

Key words: adrenal cortex, aging, ovariectomy, $17 \alpha$-hydroxylase, $\mathrm{C}_{17-20}$ lyase

\section{Introduction}

Aging is associated with many functional changes, e.g., in the cardiovascular system, in bone and lipid metabolism, and in endocrine organs. The effects of estrogen deficiency in postmenopausal women on various tissues has been well documented. In postmenopausal women, the adrenal cortex is believed to be the only source of peripheral sex hormones. Peripheral serum levels of dehydroepiandrosterone sulfate (DHEA-S), the main adrenal androgen, starts to gradually decline in the $20 \mathrm{~s}$ and $30 \mathrm{~s}$; however, there is little change in cortisol levels despite a lack of changes of adrenocorticotropin (ACTH) secretion with age. The discrepancy between levels of adrenal androgens and cortisol and the control mecha-

Department of Obstetrics and Gynecology, Showa Univcrsity School of Medicine, 1-5-8 Hatanodai, Shinagawa-ku, Tokyo 142, Japan. 
nisms of adrenal androgen secretion have been discussed ${ }^{1-3)}$. Vermeulen et al. reported the response of plasma levls of $\mathrm{C}_{19}$ and $\mathrm{C}_{21}$ steroids on acute ACTH stimulation in young and elderly men and women ${ }^{4}$. They sugested that the maintenance or increase of $\Delta 4$ steroid secretion in response to ACTH stimulation in elderly persons despite decreases of response of all 45 -steroids might due to the preservation of $3 \beta$-hydroxysteroid dehydrogenase ( $3 \beta$-HSD) activity for acute cortisol secretion. Results of our previous study suggest decreases of both $\Delta 5$ and 44-androgens, DHEA and androstenedione dione (A), in response to ACTH with preservation of the response of $17 \alpha$-hydroxyprogesterone (17-P) in postmenopausal women strengthens the physiologic role of 17-P. The results also suggest an age-associated decrease in $\mathrm{C}_{17-20}$ lyase, which catalyzes the transformation of $17 \alpha$-hydroxylated $\mathrm{C}_{21}$ steroid to the corresponding $\mathrm{C}_{19}$ steroid. However, $3 \beta$-HSD activity does not decrease, since $3 \beta$-HSD activity is influenced by estrogen in various tissues and removal of estrogen inhibition by menopause maintains $3 \beta$-HSD activity in the adrenal cortex.

Although the decline in ovarian function in postmenopausal women causes a marked decrease in estrogen production, the influence of ovarian steroids on adrenal androgen production is not fully understood. The postmenopausal ovary still produce androgens ${ }^{5)}$; however, previous studies of age-related changes of adrenocortical function did not exclude the ovarian contribution ${ }^{4,6)}$. Therefore, the present study was designed to clarify a possible effect of the ovary on adrenal steroid secretion in response to ACTH stimulation by comparing the response of peripheral steroid levels after ovariectomy in young and elderly women.

\section{Subjects and Methods}

\section{Subjects}

The subjects were reproductive-age women with regular menstruation (mean age, 33.0 years [range, 29-39 years], $n=6$ ) and postmenopausal women (mean age, 63.3 years [range, 52-76 years], $\mathrm{n}=9$ ) scheduled to undergo hysterectomy with bilateral adnexectomy for cervical cancer. Reproductive-age women were endocrinologically healthy, and postmenopausal women were at least 5 years past menopause. Peripheral blood was collected before and 2 weeks after the operation, and steroid levels were measured to compare the effect of the ovary on peripheral steroid concentrations with age. Two weeks after ovariectomy, the rapid ACTH stimulation test ${ }^{6)}$ was performed in the same patients to evaluate age-related changes in adrenocortical response to ACTH without the influence of ovarian function. Tetracosactide acetate (Cortrosyn ${ }^{\circledR}$, Daiichi Pharmaceutical Co., Tokyo) was given intravenously, and blood samples wer obtained before and 30 minutes after the injection. Serum samples were kept frozen at $-40^{\circ} \mathrm{C}$ until assayed. The purpose of the study was explained and informed consent was obtained from all patients.

\section{Steroid measurements}

Peripheral concentrations of pregnenolone $\left(P_{5}\right), 17 \alpha$-hydroxypregnenolone $\left(17-P_{5}\right)$, progesterone (P), 17-P, DHEA, A, and cortisol were measured by radioimmunoassay with specific antibodies and chromatographic separation with a Sephadex LH-20 microcolumn as previously described ${ }^{6,7)}$.

\section{Statistical analysis}

Student's $t$-test and paired $t$-test were used for statistical analysis. 
Table 1. Steroid levels before and 2 weeks after ovariectomy in reproductive-age and postmenopausal women.

\begin{tabular}{lccccc}
\hline \multirow{2}{*}{ Steroids $(\mathrm{ng} / \mathrm{ml})$} & \multicolumn{2}{c}{ Reproductive-age wemen $(\mathrm{n}=6)$} & & \multicolumn{2}{c}{ Postmenopausal women $(\mathrm{n}=9)$} \\
\cline { 2 - 3 } & before & after & & before & after \\
\hline Pregnenolone & $1.02 \pm 0.12$ & $0.53 \pm 0.07^{*}$ & & $0.68 \pm 0.09$ & $0.64 \pm 0.06$ \\
Progesterone & $0.73 \pm 0.04$ & $0.44 \pm 0.08^{*}$ & & $0.49 \pm 0.04$ & $0.42 \pm 0.04$ \\
$17 \alpha$-hydroxypregnenolone & $1.36 \pm 0.11$ & $0.76 \pm 0.09^{*}$ & & $0.70 \pm 0.05$ & $0.67 \pm 0.05$ \\
$17 \alpha$-hydroxyprogesterone & $0.65 \pm 0.08$ & $0.22 \pm 0.01^{*}$ & & $0.41 \pm 0.05$ & $0.30 \pm 0.02$ \\
Dehydroepiandrosterone & $3.89 \pm 0.36$ & $1.84 \pm 0.13^{*}$ & & $1.97 \pm 0.24$ & $1.68 \pm 0.15$ \\
Androstenedione & $0.66 \pm 0.05$ & $0.39 \pm 0.03^{*}$ & & $0.45 \pm 0.02$ & $0.40 \pm 0.03$ \\
\hline
\end{tabular}

before vs after (ovariectomy), ${ }^{*} \mathrm{p}<0.01$, mean \pm S.E.
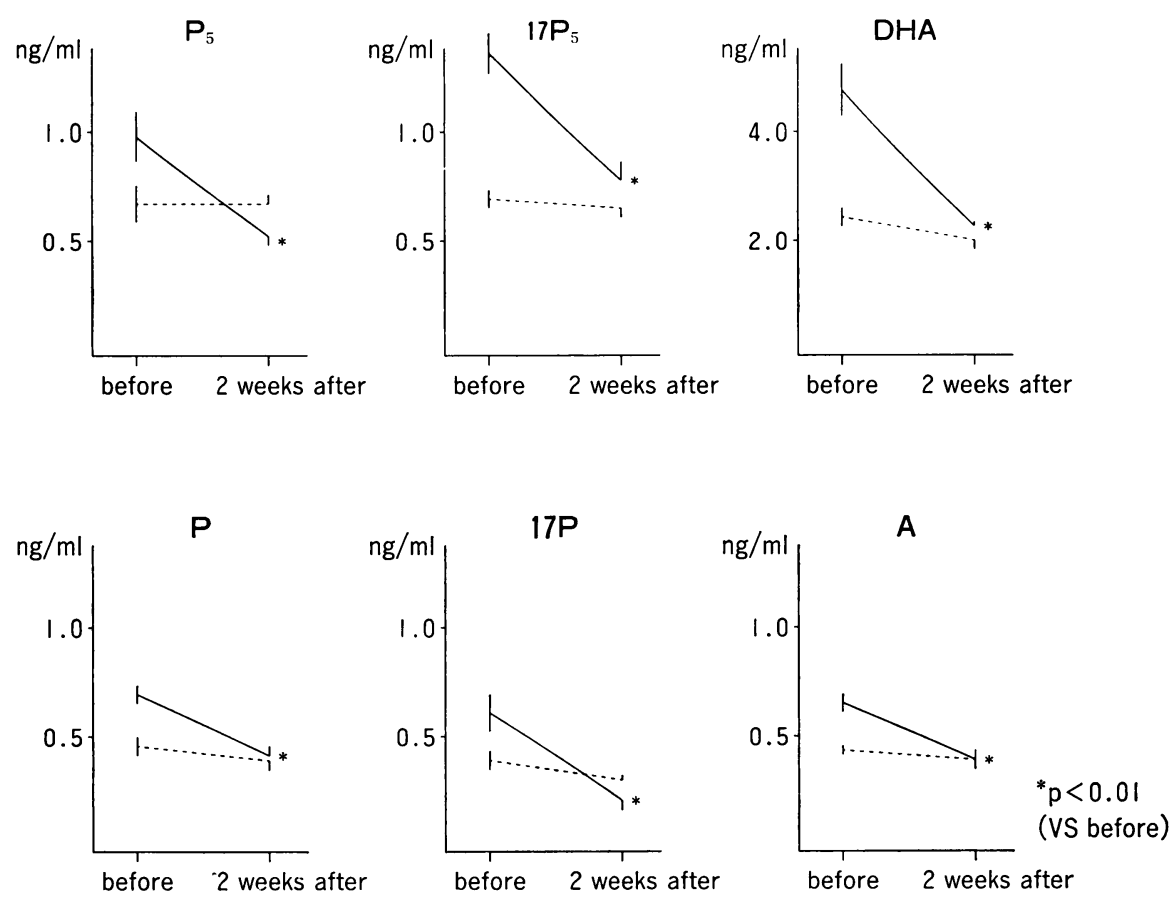

$$
\begin{array}{ll}
\text { - reproductive-age } n=6 \quad \text { Mean } \pm \text { S.E. } \\
\text { … }
\end{array}
$$

Fig. 1. Changes of steroid levels before and 2 weeks after ovariectomy. Peripheral steroid levels were measured in ovariectomized reproductive and menopausal women by radioimmunoassay.

\section{Results}

\section{Changes in steroid levels before and after ovariectomy with age}

Changes in steroid levels before and 2 weeks after ovariectomy in reproductive-age and postmenopausal women were determined (Table 1). Significantly higher concentrations of $\mathrm{C}_{21}$ steroids $\mathrm{P}_{5}, \mathrm{P}, 17-\mathrm{P}_{5}$, and 17-P and $\mathrm{C}_{19}$ steroids DHEA and $\mathrm{A}$ were observed in re- 


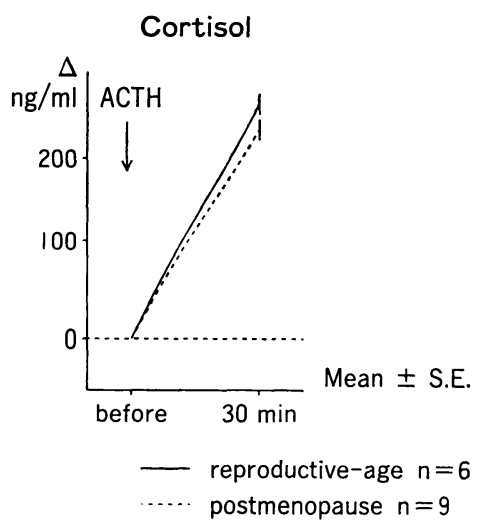

Fig. 2. Stimulating effect of ACTH on peripheral cortisol levels in women 2 weeks after ovariectomy. Acute ACTH stimulation test (cortrosyn ${ }^{\oplus} 0.25 \mathrm{mg}$ i.v.) was performed to reproductive $(n=6)$ and menopausal $(n=9)$ women who were ovariectomized. Peripheral venous blood were collected before and 30 minutes after ACTH administration. No significant change of the increment of overbasal levels in cortisol between reproductive and menopausal women was observed.

productive-age women. Steroid levels were significantly $(\mathrm{p}<0.01)$ decreased to the levels of postmenopausal women by ovariectomy (Fig. 1). On the other hand, no significant changes were obtained before and after ovariectomy in any of the steroids in postmenopausal women.

Response of steroid levels to ACTH stimulation of different ages in ovariectomized women

The rapid ACTH stimulation test was performed in the same patients who underwent ovariectomy 2 weeks earlier, and the differences in response of steroid levels to ACTH stimulation between reproductive-age and postmenopausal women were compared. Increases over basal levels in response to ACTH stimulation were observed (Fig. 2 and 3). There was no difference in the response of cortisol levels to ACTH between reproductive-age and postmenopausal women. No age-associated change in $\mathrm{P}_{5}, 17-\mathrm{P}_{5}, \mathrm{P}$, or 17-P in response to ACTH stimulation was shown. On the other hand, significant decreases in the responses of DHEA and A to ACTH stimulation in postmenopausal women were observed.

\section{Discussion}

Cortisol secretion from the adrenal cortex in response to stress via the hypothalamicpituitary axis is maintained despite increased age. Basal plasma concentrations of glucocorticoids, measured as immunoreactive cortisol, tend not to change with age, despite a corresponding decrease in the metabolic clearance of cortisol $^{8}$. Neither the amount nor the binding of corticosteroid-binding globulin (CBG) decreases significantly with age ${ }^{9}$. Direct stimulation of the adrenal cortex by ACTH results in normal or slightly prolonged cortisol response in elderly people ${ }^{8)}$. In contrast to the minimal effects on cortisol secretion, levels of peripheral $\mathrm{C}_{21}$ and $\mathrm{C}_{19}$ steroids, including 17- $\mathrm{P}_{5}, 17-\mathrm{P}$, DHEA, and A, decrease significantly in postmenopausal women ${ }^{6}$. These steroids are produced by both the adrenal cortex and the ovary, and the concentrations of these steroids in the ovarian vein in postmenopausal women are significantly higher than in peripheral veins ${ }^{5,10)}$. Greenblatt et al. reported 
$P_{5}$

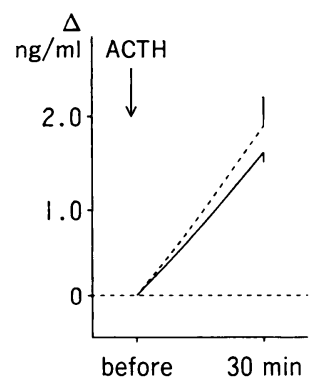

P

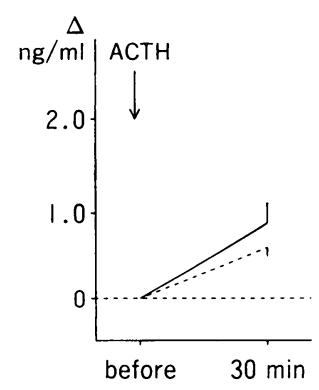

$17 \mathrm{P}_{5}$

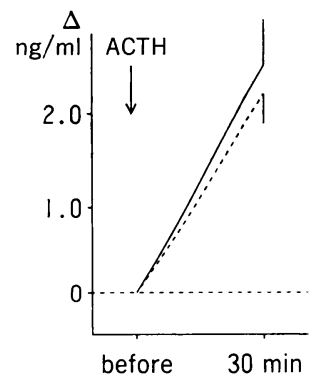

17P

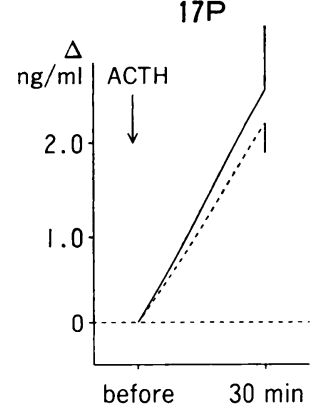

DHA

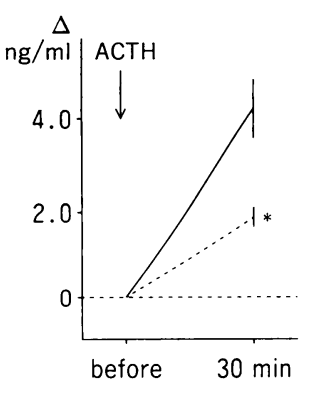

A

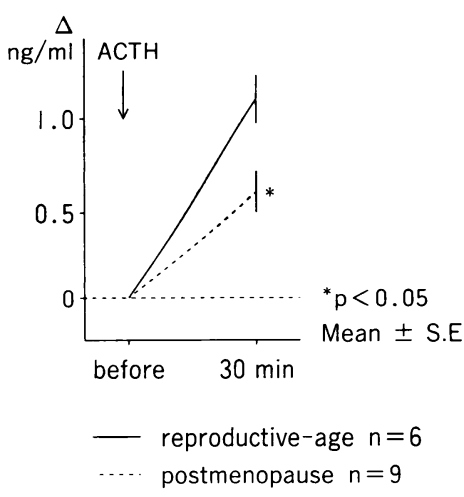

Fig. 3. Stimulating effect of ACTH on peripheral steroid levels in women 2 weeks after ovariectomy. Acute ACTH stimulation test (cortrosyn ${ }^{\circledR} 0.25 \mathrm{mg}$ i.v.) was performed to reproductive $(n=6)$ and menopausal $(n=9)$ women who were ovariectomized. Peripheral venous blood were collected before and 30 minutes after ACTH administration.

that the postmenopausal ovary produces androgens but no estrogens in response to intravenous administration of human chorionic gonadotropin (hCG) ${ }^{5)}$. According to our previous report, steroid concentrations in ovarian venous blood of postmenopausal women were elevated when the acute ACTH stimulation test was performed during gynecologic operations and steroids from the adrenal cortex were elevated in peripheral veins ${ }^{10)}$. The results support the hypothesis that the postmenopausal ovary participates in the response to peripheral changes in steroid levels.

To our knowledge, this is the first report of age-related changes in adrenocortical steroidogenesis without the influence of ovarian function in women who had undergone ovariectomy. First, the differences in the values of peripheral steroids before and after ovariectomy in reproductive-age and postmenopausal women were demonstrated. The lack of difference in values before and after ovariectomy in postmenopausal women suggests that the ovarian contribution to peripheral steroid levels in postmenopausal women is limited. Therefore, the difference in the response of peripheral steroid levels to acute ACTH stimulation with age was determined. No significant difference in peripheral levels of $\mathbf{P}_{5}, \mathbf{P}, 17-\mathbf{P}_{5}$, or 17-P in response to ACTH stimulation between reproductive-age and postmenopausal women was 
observed. Furthermore, a marked response of $17 \alpha$-hydroxylated $\mathrm{C}_{21}$ steroids to ACTH was also obtained. However, it is of interest that the response of $\mathrm{C}_{19}$ steroids in postmenopausal women was significantly lower than that in reproductive-age women. Results of the present study indicate the preservation of adrenocortical $17 \alpha$-hydroxylase activity and a reduction of $\mathrm{C}_{1 i-20}$ lyase activity in postmenopausal women. These findings are in agreement with those of a previous review of the divergence of adrenal androgen and cortisol ${ }^{2}$. Kamiyama et al. measured enzyme activities of the postmenopausal ovary by in vitro incubation and found a significant decrease in $\mathrm{C}_{17-20}$ lyase but not in $17 \alpha$-hydroxylase activity of the postmenopausal ovary ${ }^{11)}$. Many reports have documented the dissociation of adrenal androgen and cortisol secretion with age ${ }^{12)}$. Since a single enzyme, P450c17, can catalyze both $17 \alpha-$ hydroxylase and $\mathrm{C}_{1 i-20}$ lyase reactions, induction of this enzyme is believed to control adrenal androgen secretion ${ }^{13-16)}$. Both DHEA production and P450c17 mRNA expression by the bovine adrenal cortex are less in old animals than in young animals ${ }^{15)}$. It is known that the testes, a major production site of androgen, possesses more potent $\mathrm{C}_{17-20}$ lyase activity than does the adrenal cortex. Results of the current report suggest that the difference in $\mathrm{C}_{17-20}$ lyase activity in various tissues and between species might be due to the regulation of electron transport for P450c17 enzyme cytochrome b5 ${ }^{16,17)}$.

Recently, estrogen replacement therapy for postmenopausal women has been widely accepted to prevent osteoporosis and cardiovascular disease. Effects of estrogen withdrawal and replacement therapy on adrenocortical steroidogenesis remain to be elucidated.

\section{References}

1) Adams JB: Control of secretion and function of $\mathrm{C}_{19}-\Delta 5$-steroids of the human adrenal gland. Mol Cell Endocrinol, 41: 1-17 (1985)

2) McKenna TJ and Cunningham SK: The control of adrenal androgen secretion. J Endocrinol, 129: 1-3 (1991)

3) Parker L, Gral T, Perrigo V and Skowsky R: Decreased adrenal andregen sensitivity to ACTH during aging. Metabolism, 30: 601-604 (1981)

4) Vermeulen A, Deslypere JP, Schelfhout W, Verdonck L and Rubens R: Adrenocortical function in old age: Response of acute adrenocorticotropin stimulation. J Clin Endocrinol Metab, 54: 187-191 (1982)

5) Greenblatt RB, Michell F and Mahesh VB: Ovarian and adrenal steroid production in the postmenopausal woman. Obstetrics and Gynecology, 47: 383-387 (1976)

6) Akamatsu T, Chiba H, Kamiyama H, Hirose K, Saito $H$ and Yanaihara T: Menopause related changes of adrenocortical steroid production. Asia-Oceania J Obstet Gynecol, 18: 271-276 (1992)

7) Hirato $K$, Yanaihara $T$, Araki $H$ and Nakayama $T$ : Serum steroid hormone levels in neonates born from the mother with placental sulfatase deficiency. Endocrinol Japon, 37: 731-739 (1990)

8) Blichert-Toft $M$, Blichert-Toft $B$ and Kaalund-Jensen H: Pituitary-adrenocortical stimulation in the aged as reflected in levels of plasma cortisol and compound S. Acta Chir Scand, 136: 665-670 (1970)

9) Gregerman RI and Bierman EL: Aging and hormones. In Textbook of Endocrinology (ed 5), Williams RH (ed): WB Saunders, pp 1192-1212 (1981)

10) Saito H, Akamatsu $T$, Kamiyama $H$, Chiba $H$, Hirato $K$ and Yanaihara $T$ : Changes in the secretion of sex steroid from adrenal cortex and ovary with age. Sanfu no Sekai, 13: 205-211 (1991) (in Japanese)

11) Kamiyama H, Hirato K, Akamatsu T, Saito H, Yanaihara T and Kushima M: Changes in steroid enzyme activities iwt hage in human ovary. Acta Obstet Gynaec Jpn, 44: 419-426 (1992)

12) Parker LN: Adrenal androgens. In Endocrinology (volume 1), DeGroot LJ (ed): W.B. Saunders Philadelphia, 1837-1850 (1995)

13) Zuber MX, Simpson ER and Waterman MR: Expression of bovine 17 $\alpha$-hydroxylase cytochrome P450 cDNA in nonsteroidogenic (COS) cells. Science, 234: 2475-2482 (1986) 
14) Couch RM, Muller J and Winter JSD: Regulation of the activities of 17-hydroxylase and 17,20desmolase in human adrenal cortex: Kinetic analysis and inhibition by endogenous steroids. J Clin Endocrinol Metab, 63: 613-618 (1986)

15) Ogo A, Haji M, Ohashi $\mathbf{M}$ and Nawata H: Decreased expression of cytochrome P450 17 $\alpha$-hydroxylase mRNA in senescent bovine adrenal gland. Gerontology, 37: 262-271 (1991)

16) Sasaki Y, Yanase T, Hara T, Takayanagi R, Haji $M$ and Nawata H: Mechanism of abnormal production of adrenal androgens in patients with adrenocortical adenomas and carcinomas. $J$ Clin Endocrinol Metab, 78: 36-40 (1994)

17) Yanagibashi $\mathrm{K}$ and Hall PF: Role of electron transport in the regulation of the lyase activity of $\mathrm{C}_{21}$ side-chain cleavage $\mathrm{P} 450$ from porcine adrenal and testicular microsome. $J$ Biol Chem, 261: 8429-8433 (1986)

18) Katagiri M, Kagawa $N$ and Waterman MR: Role of cytochrome b5 in the biosynthesis of androgens by human P450c17. Achiv Biochem Biophys, 317: 343-347 (1995)

[Received November 18, 1995: Accepted November 30, 1995] [Express] 